\title{
ARTEMISININ AND TOTAL FLAVONOID CONTENT IN IN VITRO CULTIVATED ARTEMISIA ANNUA L. "HAIRY" ROOT CULTURE
}

\section{Drobot Kateryna ${ }^{1 *}$, Matvieieva Nadiia ${ }^{1}$, Kharkhota Maxim², Shakhovsky Anatolii ${ }^{1}$, Ratushnyak Yakiv ${ }^{1}$}

\author{
${ }^{1}$ Institute of Cell Biology and Genetic Engineering, NAS of Ukraine, Kyiv, Ukraine \\ ${ }^{2}$ D.K. Zabolotny Institute of Microbiology and Virology, NAS of Ukraine, Kyiv, Ukraine
}

Received 20. 6. 2017

Revised 22.6. 2017

Published 27.11.2017

\begin{abstract}
In this paper artemisinin and total flavonoid content in in vitro cultivated non-transformed Artemisia annua L. plants and "hairy" root culture were compared. The amount of total flavonoids was determined using modified Aluminium chloride colorimetric method. Determination of artemisinin in Artemisia annua extracts was carried out using HPLC analysis. Transgenic roots of Artemisia annua accumulated flavonoids in more degree than the roots (1.8 times higher) and leaves (1.6 times higher) of in vitro cultivated control plants. At the same time the control roots synthesized artemisinin four times more (up to $6.5 \mathrm{mg} / \mathrm{g}$ dry weight) than "hairy" roots. Owing to the higher growth rate (almost 40 times higher in 3 weeks of cultivation) transgenic roots may be suitable for artemisinin production. The data obtained suggest the possibility of using of Artemisia annua "hairy" root culture as a potential source of flavonoids and artemisinin.
\end{abstract}

Keywords: Artemisia annua L.; in vitro cultivation; “hairy” root culture; artemisinin; flavonoids

\section{Introduction}

It is known that oxidative stress is one of the essential factors of aging and development of degenerative diseases. Free radicals, generated under oxidative stress conditions are able to oxidize biomolecules and may cause DNA damage and lipid peroxidation. These processes initiation may results in mutagenesis and cell death (Lobo et al., 2010). Natural antioxidants, such as flavonoids, help to prevent oxidative stress induced deterioration (Cotelle, 2001). Plants are an effective source of natural antioxidants. However, plants and herbs vary in their antioxidant capacity, because of species, varieties, growth conditions, genetics, etc.

Sweet wormwood (Artemisia annua L.) - belongs to Asteraceae family and is native to temperate Asia. This species was naturalized in many countries including Ukraine (Boiko, 2013). Interest to Artemisia annua plants is related to the extraction of high-efficient antimalarial compound artemisinin from the leaves of this plant (Tu, 2011). A. annua plants can be used also as a source of other biologically active compounds.

It was studied that Artemisia annua extracts possess antimicrobial, anti-inflammatory and antioxidant activities due to the presence of essential oils, phenols, flavonoids and other bioactive substances (Mueller et al., 2000). Moreover synergetic effects of flavonoids combined with artemisinin were demonstrated in cancer treatment. (Ferreira et al., 2010). Over 40 flavonoids were found in Artemisia

*Corresponding author: Kateryna Drobot, Institute of Cell Biology and Genetic Engineering, NAS of Ukraine, Kyiv, Ukraine, $\triangle$ katyadrobot@gmail.com 
annua. Eupatin, quercetin, apigenin, rutin, luteolin, kaempferol are among them. Therefore phytochemical study of sweet wormwood roots is of great interest. Biotechnological interventions may be helpful to increase the value of Artemisia annua plants (Grech-Baran and Pietrosiuk, 2012).

"Hairy" root culture is a type of plant tissue culture, which could be obtained via Agrobacterium rhizogenes-mediated transformation. It is considered to be an alternative way for producing valuable plant-derived compounds (Srivastava and Srivastava, 2007). «Hairy» root culture is characterized by unlimited and fast growth without use of exogenous hormones; it is unpretending to the growth conditions and doesn't require lighting; the roots can be cultivated in bioreactors. "Hairy» roots of many species have been reported to produce large variety of secondary metabolites native to plant from which it was derived (Christey, 2001).

The aim of this work was to determine the effect Agrobacterium rhizogenes-mediated transformation on artemisinin and total flavonoid content and to compare the content of these compounds in in vitro cultivated sweet wormwood Artemisia annua plants and "hairy" root culture.

\section{Materials and methodology}

Leaves and roots of non-transformed Artemisia annua plants cultivated in in vitro conditions on the hormone free half strength Murashige and Skoog ( $1 / 2$ MS) (Murashige and Skoog, 1962) basal medium were used for the experiments. "Hairy" roots were obtained earlier and were subcultured every two weeks on the same medium at $24^{\circ} \mathrm{C}$ and $16 \mathrm{~h} / \mathrm{d}$ photoperiod. Growth rate of the of control plants and transgenic root lines was studied after 3 weeks of cultivation of excised root tips on $1 / 2$ MS medium at the same conditions.

Plant material was collected after 1 month of cultivation, lyophilized and mashed into powder. The amount of total flavonoids in the $70 \%$ ethanolic extracts of Artemisia annua was determined using modified Aluminium chloride colorimetric method (Pękal and Pyrzynska, 2014). Rutin was used as standard, and the results were expressed as rutin equivalents (RE) in milligram per gram of dry weight.

For artemisinin determination Artemisia annua herb powder was macerated using ethyl acetate solvent in 1/40 ratio during 20 minutes. The mixture was filtered using vacuum pump. Solution was evaporated using SpeedVac Savant AES 2010 concentrator (Labconco, USA). Precipitate was dissolved in $2 \mathrm{ml}$ of acetonirtile.

Artemisinin and its co-products (ART) analysis was performed using HPLC-MS system. Chromatographic analysis was conducted using equipment of Centre of collective usage of Zabolotny Institute of Microbiology and Virology NAS of Ukraine (High-efficiency liquid chromatograph Agilent 1200, Agilent Technologies, USA). The separation was carried out by isocratic method using ZORBAX SB-C18 $2.1 \mathrm{~mm} \times 150 \mathrm{~mm}, 3.5 \mu \mathrm{m}$ (Agilent Technologies, USA) column with a $30^{\circ} \mathrm{C}$. Water with methanol and acetonitrile $(30 / 20 / 50 \mathrm{v} / \mathrm{v})$ were used as a mobile phase with $0.4 \mathrm{ml} / \mathrm{min}$ flow-rate. Diode array detector (DAD) wavelength used was set to 210, 280 and $216 \mathrm{~nm}$ respectively. The samples were analyzed separately according to their retention times. The spectrum of samples was compared with the spectrum of artemisinin standard (Sigma-Aldrich, catalog number 63968-64-9).

Statistical analysis was performed according to Student multiple range test. Differences from control values were significant at $p \leq 0.05$. Experiment was conducted in three replications. 


\section{Results and discussion}

In our study flavonoids were found out both in the roots and leaves of non-transformed Artemisia annua plants. The amount of this compound was $19.4 \pm 1.95 \mathrm{mg} / \mathrm{g}$ RE and $22.3 \pm 0.83 \mathrm{mg} / \mathrm{g}$ RE in roots and leaves respectively.

Flavonoids content in Artemisia annua "hairy" root lines varied from $27.5 \pm 0.53 \mathrm{mg} / \mathrm{g}$ RE to $35.6 \pm 1.38 \mathrm{mg} / \mathrm{g}$ RE.

All transgenic root lines accumulated flavonoids in amount greater than roots and leaves of the control plants (1.8 times higher and 1.6 times higher respectively). So, the genetic transformation has led to the increase of flavonoids content in Artemisia annua "hairy" root lines compared to the wild-type plants.

Artemisia spp. plants are known to synthesize flavonoids. Accumulation of the latter in Artemisia annua was mostly studied in the aerial parts (leaves, inflorescence) of in vivo grown plants. It was shown, that total flavonoid content may vary in a vast range depending on various factors: part of plant, ontogenesis stage, growth conditions (Baraldi et al., 2008). For example, eupatin content varied in this study from $0.035 \mathrm{mg}$ per $100 \mathrm{~g} \mathrm{DW}$ at pre-flowering stage up to $0.69 \mathrm{mg}$ per $100 \mathrm{~g} \mathrm{DW}$ after flowering. According to Skowyra et al. (Skowyra et al., 2014) rutin concentration in Artemisia annua extract reached up to $0.765 \mu \mathrm{g} / \mathrm{g}$ DW, that was much lower than reported in the literature.

Recently it was shown by Dilshad et al. (2016) that transgenic plants of Artemisia annua carrying rol $B$ gene demonstrated threefold increase in rutin and caffeic acid, sixfold increase in isoquercetin, and fourfold increase in quercetin as compared to the wild-type plants. They suggested that flavonoids content increase was caused by altering the expression level of the genes of flavonoids biosynthetic pathway. In addition, in another study (Bulgakov, 2008), rol genes in plant-Agrobacterium interactions were shown as potential activators of secondary metabolism in transformed cells from different plant families.

Artemisia annua in vitro cultivated plants synthesized ART. These compounds were found in roots of the control plants. ART content in the roots of Artemisia annua wild-type was higher than in "hairy" roots and reached up to $6.5 \mathrm{mg} / \mathrm{g}$ DW. ART content in "hairy" roots varied in the range 0.43-1.64 mg/g DW. Thus, transgenic roots accumulated artemisinin and its co-products in lower concentration compared with the control roots.

Comparison of growth rate of transgenic root lines was the next step of our investigation. It appeared that mass increase of non-transformed roots and transgenic roots was 0.005 and $0.104-0.186 \mathrm{~g}$ respectively in 3 weeks of cultivation. Due to the differences in the growth rate and artemisinin content in transgenic and control roots, transgenic roots can accumulate 10 times more artemisinin than control roots ( 0.305 and 0.0325 mg respectively) after 3 weeks of cultivation. Thus, ART accumulation in "hairy" roots of Artemisia annua was more effective due to the higher growth rate than in roots of in vitro cultivated Artemisia annua plants. It is known that "hairy" root culture is unpretending to the growth conditions and doesn't require lighting and exogenous plant hormones, what makes it a potential system for BAS production, e.g. artemisinin.

\section{Conclusions}

Our investigation approved that transgenic roots of Artemisia annua obtained via Agrobacterium rhizogenes-mediated transformation can accumulateflavonoids in more degree than non-transformed plants. Transgenic roots accumulated artemisinin and its co-products in lower concentration 
compared with the roots of in vitro cultivated sweet wormwood, but owing to the higher growth rate they are more suitable for artemisinin production.

The data obtained suggest the possibility of using Artemisia annua "hairy" root culture as a source of antioxidants. However, despite the fact that Aluminium chloride colorimetric method is reliable and widely used to determine flavonoid content in the plant material, other antioxidant mechanisms are not captured by the method. Thus, there is an urge to apply several methods to estimate antioxidant potential of extracts.

\section{Acknowledgments}

Publication is based on the research provided by the grant support of the State Fund For Fundamental Research, Ukraine (No Ф73/2-2017).

\section{References}

Baraldi, R., Isacchi, B., Predieri, S., Marconi, G., Vincieri, F.F., Bilia, A.R. 2008. Distribution of artemisinin and bioactive flavonoids from Artemisia annua L. during plant growth. Biochem Syst Ecol, vol. 36, no. 5, p. 340-348. DOI: 10.1016/j.bse.2007.11.002

Boiko, A.V. 2013. Specific features of Artemisia L. species distribution of the flora of Ukraine. Industrial Botany, vol. 13, p. 73-79. http://dspace.nbuv.gov.ua/handle/123456789/67673

Bulgakov, V.P. 2008. Functions of rol genes in plant secondary metabolism. In Biotechnol Adv, vol. 26, no. 4, p. 318-324. DOI: 10.1016/j.biotechadv.2008.03.001

Christey, M.C. 2001. Use of Ri-mediated transformation for production of transgenic plants. In Vitro Cell Dev, vol. 37, no. 6, p. 687-700. DOI: 10.1007/s11627-001-0120-0

Cotelle, N. 2001. Role of flavonoids in oxidative stress. In Curr Top Med Chem, 1(6), 569-590. DOI: $10.2174 / 1568026013394750$

Dilshad, E., Zafar, S., Ismail, H., Waheed, M.T., Cusido, R.M., Palazon, J., Mirza, B. 2016. Effect of Rol Genes on Polyphenols Biosynthesis in Artemisia annua and Their Effect on Antioxidant and Cytotoxic Potential of the Plant. In Appl Biochem Biotechnol, 179(8), 1456-1468. DOI: 10.1007/s12010-016-2077-9

Ferreira, J.F.S., Luthria, D.L., Sasaki, T., Heyerick, A. 2010. Flavonoids from Artemisia annua L. as antioxidants and their potential synergism with artemisinin against malaria and cancer. In Molecules, 15, 3135-3170. DOI: 10.3390/molecules15053135

Grech-Baran, M., Pietrosiuk, A. 2012. Artemisia species in vitro cultures for production of biologically active secondary metabolites. In BioTechnologia, 93(4), 371-380. DOI: 10.5114/bta.2012.46591

Lobo, V., Phatak, A., Chandra, N. 2010. Free radicals and functional foods: impact on human health. In Pharmacogn. Rev, 4(1), 118-126. DOI: 10.4103/0973-7847.70902

Mueller, M.S., Karhagomba, I.B., Hirt, H.M., Wemakor, E. 2000. The potential of Artemisia annua as a locally produced remedy for malaria in the tropics, agricultural, chemical and clinical aspects. In J Ethnopharmacol, 73(3), 487-493. DOI: 10.1016/S0378-8741(00)00289-0

Murashige, T., Skoog, F. 1962. A revised medium for rapid growth and bioassay with tobacco tissue culture. In Plant Physiology, 15(3), 473-497. DOI: 10.1111/j.1399-3054.1962.tb08052.x

Pękal A., Pyrzynska, K. 2014. Evaluation of aluminium complexation reaction for flavonoid content assay. In Food Analytical Methods, 7(9), 1776-1782. DOI: 10.1007/s12161-014-9814-x.

Skowyra, M., Gallego, M.G., Segovia, F., Almajano, M.P. 2014. Antioxidant Properties of Artemisia annua Extracts in Model Food Emulsions. In Antioxidants (Basel), vol. 3(1), 116-128. DOI:10.3390/antiox3010116.

Srivastava, S., Srivastava, A. K. 2007. "Hairy" root culture for massproduction of high-value secondary metabolites. In Crit Rev Biotechnol, vol. 27, p. 29-43. DOI: 10.1080/07388550601173918

Tu, Y. 2011. The discovery of artemisinin (qinghaosu) and gifts from Chinese medicine. In Nature Medicine, 17(10), 1217-1220. DOI: 10.1038/nm.2471 